\title{
Evaluation of the stability enhancement of the conventional sliding mode controller using whale optimization algorithm
}

\author{
Aws Mahmood Abdullah ${ }^{1}$, Ali Mohsin Kaittan ${ }^{2}$, Mustafa Sabah Taha ${ }^{3}$ \\ ${ }^{1}$ Avicenna E-learning Center, University of Baghdad, Iraq \\ ${ }^{2}$ Asoul Aldean University College, Baghdad, Iraq \\ ${ }^{3}$ Missan Oil Training Institute, Ministry of Oil, Iraq
}

\begin{tabular}{l} 
Article Info \\
\hline Article history: \\
Received Apr 17, 2020 \\
Revised Jun 5, 2020 \\
Accepted Jun 14, 2020 \\
\hline
\end{tabular}

\section{Keywords:}

Derivative of error

Desired position

Error

Reaching stage

Sliding stage

\begin{abstract}
The proposed work is an attempt to investigate the stability of the nonlinear system by using a whale optimization algorithm as of one of the metaheuristic optimization methods, and this investigation was conducted on a single inverted pendulum as a study model. The evaluation measures which were used in this article values of gain and sliding surface of the conventional sliding mode controller to illustrate the extent of the system`s stability. Furthermore, control action, the relationship between error and its derivative, desired, and actual position in addition to sliding response graphically showed the feasibility of the proposed solution. The attained results illustrated considerable improvement in the settling time and minimizing the impact of chattering behavior.
\end{abstract}

This is an open access article under the CC BY-SA license.

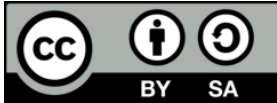

Corresponding Author:

Mustafa Sabah Taha

Missan Oil Training Institute

Ministry of Oil, Iraq

Email: timimymustafa@gmail.com

\section{INTRODUCTION}

Nowadays, internet communication becomes a significant part of the infrastructure. Based on the internet, most of the applications of infrastructure systems can be operated [1, 2]. For the past decades, power system optimization techniques have been subject to many studies for planning and strategy development [3]. Comprehensively, controlling any particular system in diverse applications can be implemented in one of two schemes model- built or non -model evolved. Model-built control schemes are considered systematic and might be conducted in conventional systems due to their qualities, which include but not limited to, reliability, precision, and different appropriate standards. But, in practical situations, more than a constraint, disturbance influencer, as well as uncertainty conditions, are changeable and not easy to represent in the model. Hence, non- model-built schemes have been exceptionally implemented because such schemes do not necessitate highly sophisticated mathematical modeling [4-6].

Based on the mentioned above, most practical systems can be varied with time due to surrounding circumstances that impact the functionality of the system; thus, the controller should be interactive with these variations. Conventional Sliding-Mode Controller (CSMCR) is an adjustable control scheme that captured large attention since the mid of the $20^{\text {th }}$ century; it represents efficient addressing for numerous control systems [7-9]. The usage of CSMCR is considerable in most nonlinear systems because it is adjustable; in other words, its configuration can be varied as the system is modified in order to obtain the required output $[10,11]$. 
Various approaches have been proposed in several research involves (CSMCR), these approaches consider the mechanism of controller design, nature of control modifying parameters in addition to characteristics of partial systems which are included in the entire system. Electrical machines such as motors and alternators; additionally, chemical procedures are handled by (CSMCR) [12, 13]. In spite of outstanding aspects of (CSMCR) which included withstanding the impact of ambiguity and external influencer, decreasing in order by one from the original system and lastly, the system controlled by (CSMCR) fulfills zero values of error and its derivative. There is a remarkable drawback that should be tackled in order to minimize the negative effect on system stability $[14,15]$; this drawback is the chattering problem as shown in Figure 1.

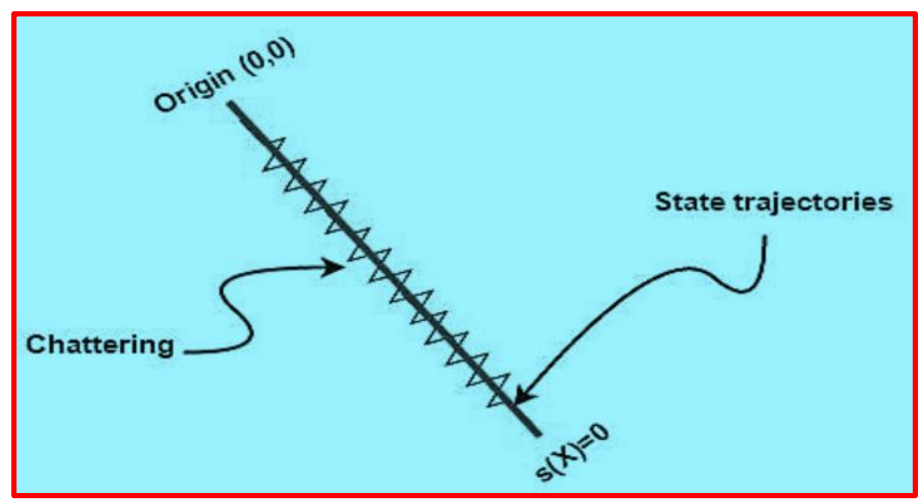

Figure 1. The chattering behavior in CSMCR

Numerous conventional methods were offered for addressing this issue; one of them is substituting the Sign mathematical function, which is considered the cause of the chattering phenomenon by another model [16, 17]. While some solutions were based on the integration between CSMCR and smart fuzzy controller [18], some of the Optimization techniques also investigated to reduce chattering impact such as genetic and particle swarm algorithms [19-23]. However; the deployment of optimization algorithms requires further investigation to illustrate the feasibility of the advanced techniques over classical mathematical models, the proposed work mainly aims to utilize a highly advanced whale optimization algorithm in order to diminish as much as possible the impact of chattering behavior and thus achieving reliable and consistent stability by finding best values of gain $G$ and the slope of sliding surface $\delta$ for (CSMCR) to ensure the stability of single inverted pendulum as a nonlinear system case study.

The main outline of this article as following; Section 2 presents problem background, including Conventional Sliding-Mode Controller, handling of chattering problem using the mathematical solution of the boundary layer, and mathematical description of the study model. Section 3 proposes the whale optimization algorithm as an effective and unconventional solution for chattering phenomena. Section 4 graphically illustrates the effect of the proposed optimization technique on the result by using control action, error, desired and actual position, and sliding response as evaluation measures. Furthermore, it computationally proves stability improvement by using gain $\mathrm{G}(\mathrm{x})$ and slope of sliding surface $\delta$ as evaluation measures. Finally, Section 5 summarizes the conclusion of this work.

\section{PROBLEM BACKGROUND}

\subsection{Conventional sliding-mode controller (CSMCR)}

The high compatibility and efficiency of the Conventional Sliding-Mode Controller push most of the designers to utilize it in order to solve the nonlinearity problem of changeable systems. The operation of CSMCR can be described into stages:

a) Reaching stage: under this phase, the trajectory gravitates to the sliding surface. Once the state trajectory reaches the sliding surface, that means the sliding stage is activated.

b) Sliding stage: under this phase the system state trajectory is necessitated to wait on the sliding surface then gliding towards the origin in limited time as shown in Figure 2. 


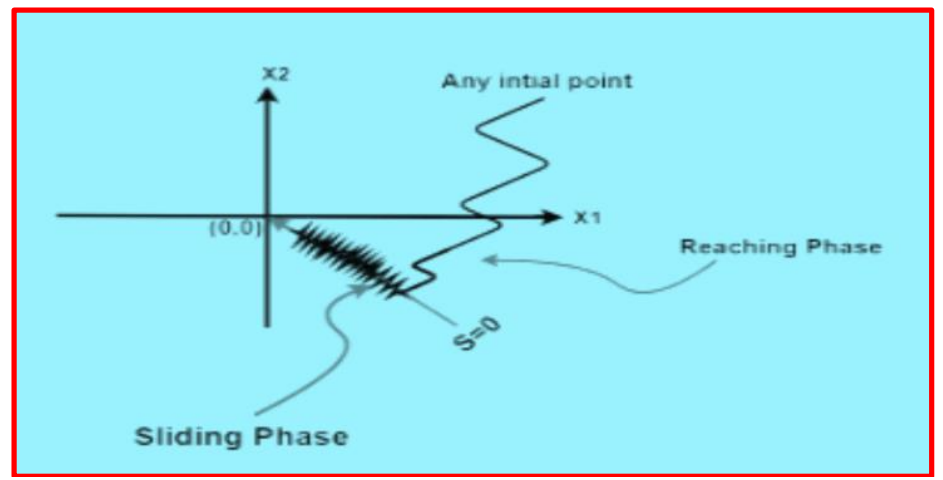

Figure 2. The two stages of the state trajectory in CSMCR

The mathematical description of the switching surface is:

$$
s s(x, t)=\delta_{e r}+e r ; \delta>\alpha
$$

where $\delta$ is a constant positive parameter, mathematically error and derivatives can be expressed as below:

$$
x_{1}=e r=\alpha-\alpha_{\text {fin }} x_{2}=e r=\alpha
$$

where $\alpha_{\text {fin }}$ is the final angle position (set point) that represents the step input. Thereby, the (1) can be rewritten as:

$$
s s(x, t)=\delta x_{1}+x_{2}
$$

when $\delta$ is equal to one, (2) will become:

$$
s s(x, t)=x_{1}+x_{2}
$$

Basically, the control formula of the Sliding-Mode Controller can be expressed as below:

$$
v=v_{\text {nom }}+v_{\text {disc }}
$$

where, $v_{\text {nom }}$ is the nominal control fragment which is working on guiding the system state trajectory toward sliding surface ( $s S=0)$, and $v_{\text {disc }}$ is the discontinuous control fragment that prerequisite in order to uphold the state trajectory nearby to the switching surface. The discontinuous control $v_{\text {disc }}$ is described below [11]:

$$
v_{\text {disc }}=-G(x) \operatorname{sign}(s s)
$$

where $G(x)$ is a discontinuous gain and $\operatorname{sig}(s)$ is a signum function Figure 3.

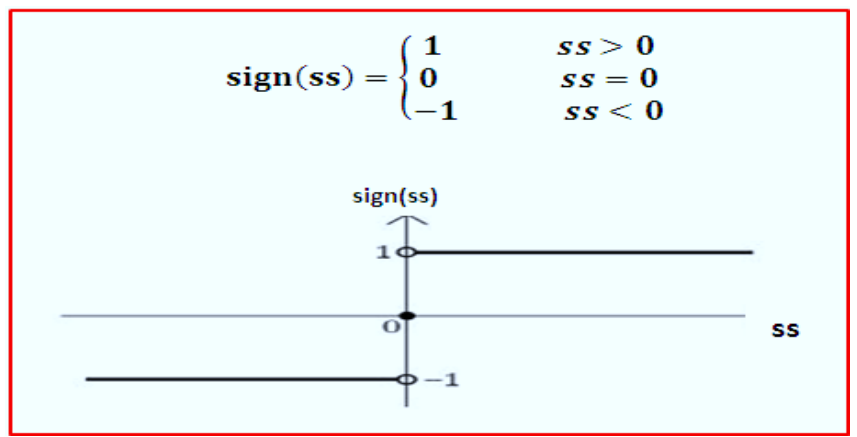

Figure 3. The mathematical pattern of the signum function 
Consequently (4) can become:

$v=v_{\text {nom }}-G(x) \operatorname{sign}(s s)$

In addition to the derivative of the sliding variable expressed as below:

$s s^{*}=x_{1}+x_{2}$

The most important thing is to uphold the $s \mathrm{~s}(x, t)$ nearby to the sliding surface. The common formula for the nonlinear system can be expressed as below:

$$
x=f(x)+B(x) u+d(x, t)
$$

With the purpose of making the right side in (7) equal to 0 , the switching gain $\mathrm{G}(x)$ should be taken mathematically as below:

$$
G(x)=G_{0}+\frac{\frac{\partial x}{\partial s s} f(x)+\frac{\partial x}{\partial s s} d(x, t)}{\frac{\partial x}{\partial s s} B(x)}
$$

The main problem that can be summarized in the existence of signum function in the discontinuous control fragment in as shown in (6) represents the cause of chattering behavior or issue, it is a noticeable downside in the sliding mode controller, and it has a negative effect on the system`s stability.

\subsection{Handling of chattering problem using mathematical solution of boundary layer}

One of the offered solutions for tackling chattering behavior is utilizing the boundary layer; the signum function is substituted by sat function as shown in Figure 4 in (6) as below:

$$
v=v_{\text {nom }}-G(x) \operatorname{sat}(s s)
$$

The mathematical expression of sat function is:

$$
\operatorname{sat}(\mathrm{ss})=\left\{\begin{array}{rr}
+1 & \text { ss } \geq+\varphi \\
\frac{\mathrm{ss}}{\varphi} & -1>\text { ss }<+1 \\
-1 & \text { ss } \leq-\varphi
\end{array} \quad \varphi\right. \text { : thickness of sat function }
$$

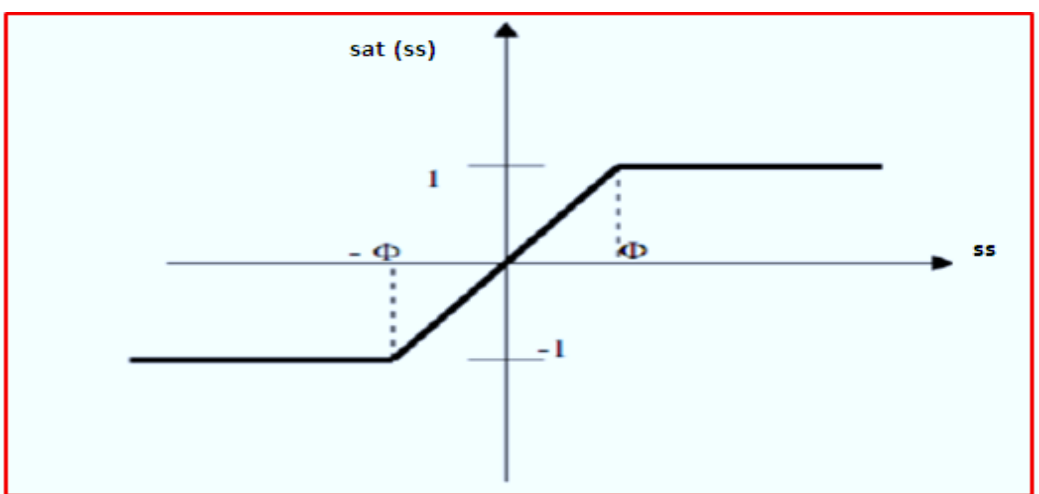

Figure 4. The mathematical pattern of sat $(\boldsymbol{s})$ function

\subsection{Study model}

As nonlinear system extensively utilized for assessing the control quality in numerous researches, a single inverted pendulum was investigated in the proposed study for controlling preciously it's position or displacement with high stability [24], the response of single inverted pendulum can be expressed mathematically as below: 
$\alpha^{\mu}=\frac{g r \sin \alpha-\left(A \times m \times \operatorname{len} \times \alpha^{2} \times \sin \alpha \times \cos \alpha\right)+A \cos \alpha v}{\operatorname{len}\left(\frac{4}{3}-A \times m \times \cos ^{2} \alpha\right)}+d(t)$

where:

$\alpha \quad$ : angular displacement (rad) of the pendulum represents the control variable or the system output

$\alpha \cdot \quad$ : angular velocity ( $\mathrm{rad} / \mathrm{second})$

$M \quad$ : pendulum cart mass $(\mathrm{kg})$

$\mathrm{m} \quad$ : pendulum mass $(\mathrm{kg})$

len : pendulum length $(\mathrm{m})$

A : constant-coefficient, and it is calculated by $(\boldsymbol{m} / \boldsymbol{M}+\boldsymbol{m})$

gr : gravity acceleration (and its value is equal to $9.8 \mathrm{~m} / \mathrm{sec}^{2}$ )

$(t)$ : exterior disturbance applied to the system

$v \quad$ : control action (in volt)

The described system experiences noticeable deviations of the above constraints. The uncertainties values of pendulum cart mass, pendulum length, and pendulum mass are $0, \pm 0.2$, and \pm 0.08 , respectively. Table 1 illustrates the nominal, least and greatest values when the parameters influenced by uncertainty.

Table 1. The constraints values due to the influence of uncertainty conditions [15]

\begin{tabular}{cccc}
\hline Constraint & Nominal & Least & Greatest \\
\hline $\mathrm{M}$ & 1 & 1 & 1 \\
len & 0.5 & 0.3 & 0.7 \\
$\mathrm{~m}$ & 0.2 & 0.12 & 0.28 \\
\hline
\end{tabular}

The error in displacement between the preferred and the real angle of the single inverted pendulum system can be expressed in the below equation:

Assume the error of displacement is $x_{1}=e r=\alpha-\alpha_{\text {fin }}$

$\alpha_{\text {fin }}:$ reference displacement, and it represents a step input

Assume the angular velocity error is taken as

$x_{2}=e^{*}=\alpha-\alpha_{\text {fin }}=\alpha \quad$ where $\alpha_{\text {fin }}=0$

Due to $\alpha_{\text {fin }}$ is constant the above-mentioned equation is expressed as below:

$x_{1}=x_{2}$

$$
x_{2} \cdot=\frac{g r \sin \left(x_{1}+\alpha_{f i n}\right)-\left(A \times m \times \operatorname{len} \times\left(x_{1}+\alpha_{f i n}\right)^{2} \times \sin \alpha \times \cos \left(x_{1}+\alpha_{f i n}\right)\right)+A \cos \left(x_{1}+\alpha_{f i n}\right) v}{\operatorname{len}\left(\frac{4}{3}-A \times m \times \cos ^{2}\left(x_{1}+\alpha_{f i n}\right)\right)}+d(t, v)
$$

In order to attain switching, gain $\mathrm{G}(x)$. It is necessitated that the derivative of the sliding mode variable equal to zero

$$
s s^{*}=x_{1}+x_{2}
$$

by replacing (12), (13) and (5) in (14),

$G(x)=G_{0}+\frac{-\left|x_{2}\right| \operatorname{maxlen}\left(4 / 3-A \operatorname{maxm}+g r-A \operatorname{maxlen}\left(x_{2}+\alpha_{f i n}\right)^{2}+\mathrm{d}(\mathrm{t}) \operatorname{maxlen}\right.}{A} \frac{\mathrm{d}(\mathrm{t}) \operatorname{maxlen}(4 / 3-A \operatorname{maxm})}{A}$

In order to design Conventional Sliding-Mode Controller for Single Inverted Pendulum, (6) is expressed into below form:

$v=\frac{-x_{2}-g r \sin \left(x_{1}+\alpha_{f i n}\right)-\left(A \times m \times l e n \times\left(x_{1}+\alpha_{f i n}\right)^{2} \times \sin \alpha \times \cos \left(x_{1}+\alpha_{f i n}\right)\right)+A \cos \left(x_{1}+\alpha_{f i n}\right) v}{\operatorname{len}\left(\frac{4}{3}-A \times m \times \cos ^{2}\left(x_{1}+\alpha_{f i n}\right)\right)}-G(x) \operatorname{sign}(s s)$

Similarly, with using sat function the equation, 10 will be expressed as below:

$v=\frac{-x_{2}-g r \sin \left(x_{1}+\alpha_{f i n}\right)-\left(A \times m \times l e n \times\left(x_{1}+\alpha_{f i n}\right)^{2} \times \sin \alpha \times \cos \left(x_{1}+\alpha_{f i n}\right)\right)+A \cos \left(x_{1}+\alpha_{f i n}\right) v}{\operatorname{len}\left(\frac{4}{3}-A \times m \times \cos ^{2}\left(x_{1}+\alpha_{f i n}\right)\right)}-G(x) \operatorname{sat}($ ss $)$ 


\section{THE PROPOSED METHOD}

\subsection{Introduction}

Today, Meta-heuristic optimization algorithms have been the most common tools in numerous engineering problems due to these algorithms depend on relatively simple perceptions, and they are applicable easily. One of these algorithms is the Whale Optimization Algorithm, which is created on the hunting method of baleen whale (WOA). This unique chasing process is entitled bubble-net feeding strategy. Whales try to catch miniature fishes nearby the sea surface by a loop path as shown in Figure 5.

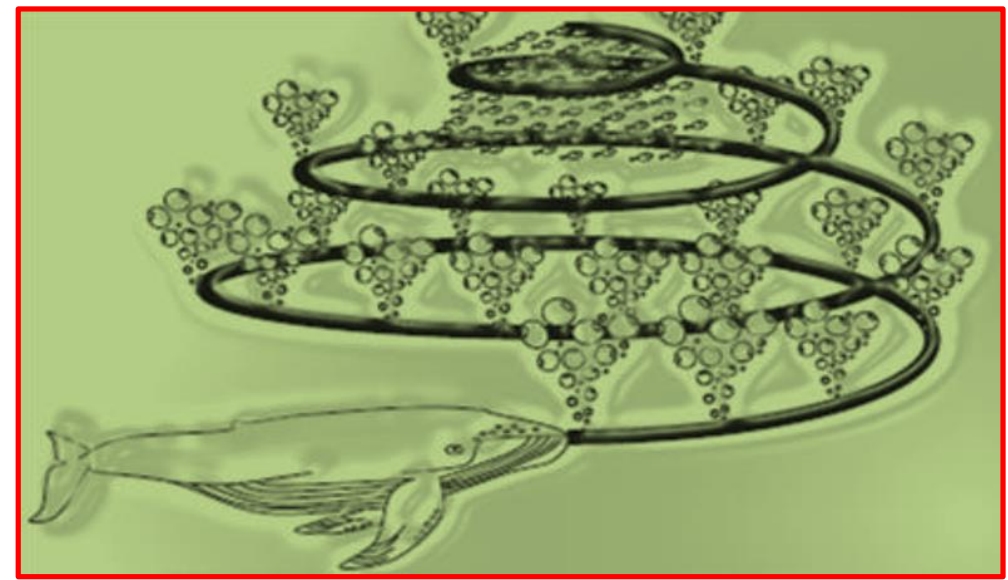

Figure 5. Bubble-net feeding technique of humpback whales

Two activities are associated with the above-mentioned conduct called upward-spirals and doubleloops. In upward-spirals, whales dive at deep $12 \mathrm{~m}$ and instigate to produce bubbles in order to surround prey, and then they swim toward the surface. Whereas double- loops include three steps coral loop, lobtail, and capture loop [25-29].

\subsection{Mathematical description}

The WOA attempts to calculate the current best candidate solution is the objective prey or is close the best. After the best search agent is determined, other search operators will successively attempt to update their positions towards the best chase agent [30, 31]. This behavior is explained by:

$$
\begin{aligned}
& D=\left|C \cdot X^{*}(t)-X(t)\right| \\
& X(t+1)=X^{*}(t)-A . D
\end{aligned}
$$

where

t : current iteration

A, C : coefficient vectors

$\mathrm{X}^{*} \quad$ : position vector of the best solution attained

$\mathrm{X}$ : the position vector

I| : absolute value and is a component-by-component multiplication

Updating of $\mathrm{X}^{*}$ is being performed at every iteration if there is a better solution. The vectors A and $\mathrm{C}$ are calculated by:

$$
\begin{aligned}
& A=2 a \cdot r-a \\
& C=2 \cdot r
\end{aligned}
$$

where

a : directly lessened from 2 to 0 throughout the duration investigation and exploitation stages

$\mathrm{r}:$ : an arbitrary vector in $[0,1]$

As shown in (18.2) authorizes all hunt operators to update their positions in the zone of the current best solution and restructures surrounding the prey.

Evaluation of the stability enhancement of the conventional sliding mode .... (Aws Mahmood Abdullah) 


\subsubsection{Bubble-net attacking strategy (exploitation phase):}

Two strategies are exploited to figure the feeding behavior of humpback whales as below:

a) Shrinking circling system:

This manner is achieved by diminishing the estimation of an in the (18.3). Moreover, the fluctuation scope of A is diminished by a. Intrinsically A is a random value in the period [-a, a] where $\mathrm{a}$ is lessened from 2 to 0 as above explained. Setting $A$ in $[-1,1]$, the new position of an inquiry operator can be considered anywhere in the middle of the first position of the specialist and the position of the current best specialist as shown in Figure 6(a).

b) Spiral redesigning position:

Figure 6(b), this method specifies parting between the whale positioned at $(\mathrm{X}, \mathrm{Y})$ and prey positioned at $\left(\mathrm{X}^{*}, \mathrm{Y}^{*}\right)$. Then winding condition is completed between the location of whale and prey to reflect the helix-molded advance of humpback whales as:

$$
\mathrm{X}(\mathrm{t}+1)=\mathrm{D}^{\prime} \cdot \mathrm{e}^{\mathrm{bl}} \cdot \cos (2 \pi \mathrm{l})+\mathrm{X} *(\mathrm{t})
$$

where $D^{\prime}=|X *(t)-X(t)|$ and determines the parting of the $i^{\text {th }}$ whale to the prey,

$\mathrm{b} \quad$ : consistent for portraying the state of the logarithmic winding

1 : random number in $[-1,1]$

Swimming of humpback whales around the prey is inside a narrowing circle and alongside a winding formed way. In order to represent this simultaneous behavior mathematically, assuming there is a probability of half to choose between either the narrowing surrounding component or the winding form to renovate the position of whales amid rearrangement as shown in two below equations.

$$
\begin{array}{ll}
X(t+1)=X^{*}(t)-A . D & \text { if } p<0.5 \\
X(t+1)=D^{\prime} \cdot e^{b l} \cdot \cos (2 \pi l)+X^{*}(t) & \text { if } \mathrm{p} \geq 0.5
\end{array}
$$

where $\mathrm{p}$ is an arbitrary number in $[0,1]$.

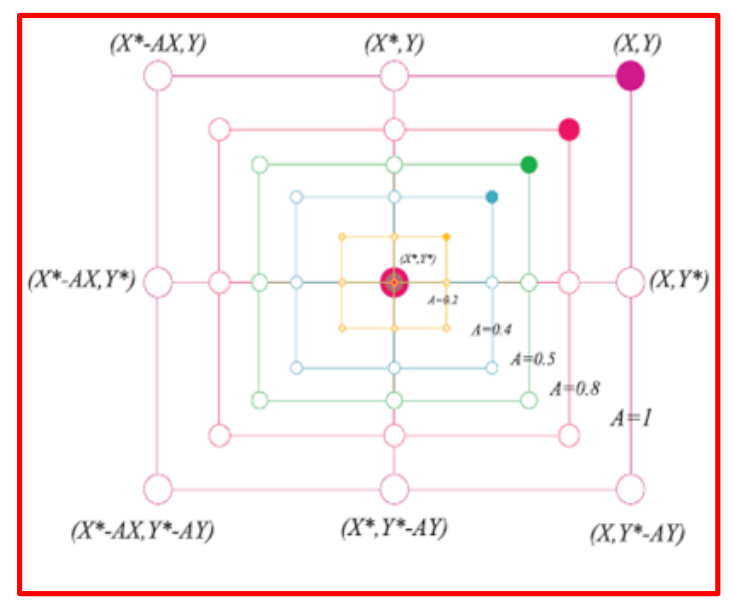

(a)

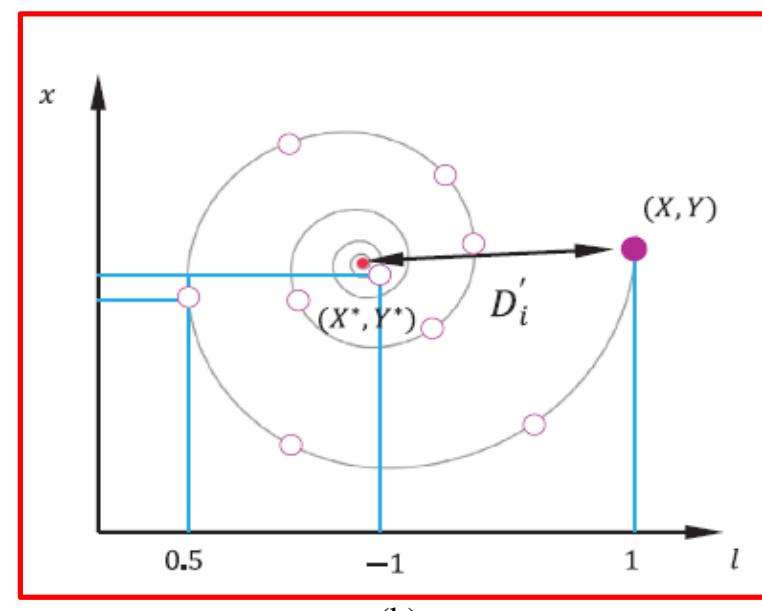

(b)

Figure 6. (a) Shrinking encircling mechanism, (b) Spiral updating position

\subsubsection{Probe for prey (investigation phase)}

Certainly, whales look for randomly according to the position of each other. Under this way, A is employed with the asymmetrical qualities greater than 1 or below -1 so as to direct the search agent to transfer away from a reference whale. Rather than the exploitation phase, the position of an inquest operator has been updated in the investigation stage according to an arbitrarily chosen search agent rather than the best chase operator exposed in this way. $|\mathrm{A}|$ greater than 1 focuses examination and accepts the WOA calculation to complete a global chase. Mathematically the procedure can be described as below:

$$
\mathrm{D}=\mid \mathrm{C} . \mathrm{X} \text { rand }-\mathrm{X} \mid
$$




$$
\mathrm{X}(\mathrm{t}+1)=\mathrm{X} \text { rand }-\mathrm{A} \cdot \mathrm{D}
$$

In the proposed work, WOA was employed in order to find best search agent $\left(\mathrm{X}^{*}\right)$, which is leading to computing optimal or close to optimal values of gain $G(x)$ and slope of sliding surface $\delta$ of (CSMCR) and thereby achieving high stability for a single inverted pendulum. The comprehensive procedure of WOA in the proposed work can be summarized in the Figure 7.

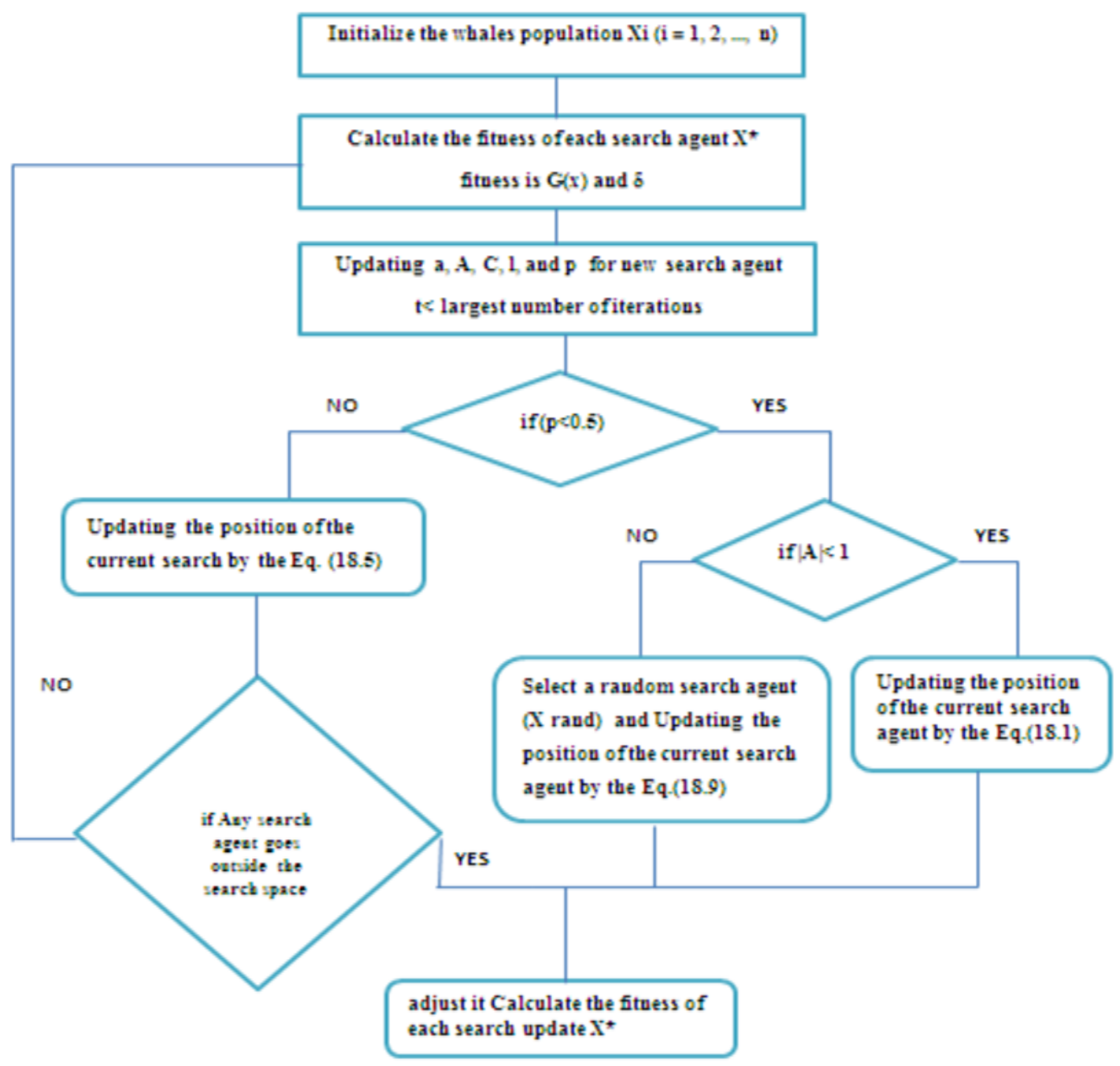

Figure 7. The comprehensive procedure of WOA in the proposed work

It is worthy of mentioning that the number of search agent and the most significant number of iterations was 30 and 300 respectively, as well as considering mentioned above highest values of uncertainty condition. Additionally, disturbance with a value of 0.4. Additionally, the proposed investigation was conducted using Matlab 7.8 version on a laptop of Intel processor, CPU 350@ $2.27 \mathrm{GHz}$, RAM 4 GB (2.99GB usable), operating system 64 bit.

\section{RESULT AND DISCUSSION}

\subsection{CSMCR with sign function}

The control action, the relationship between error and its derivative, desired and actual position as well as sliding response have been illustrated in Figure 8(a)-(d). 


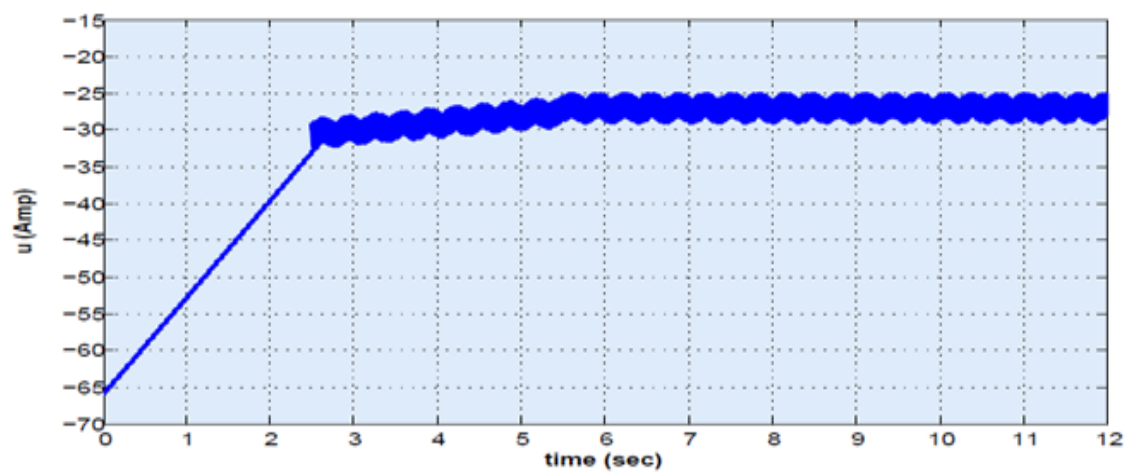

(a)

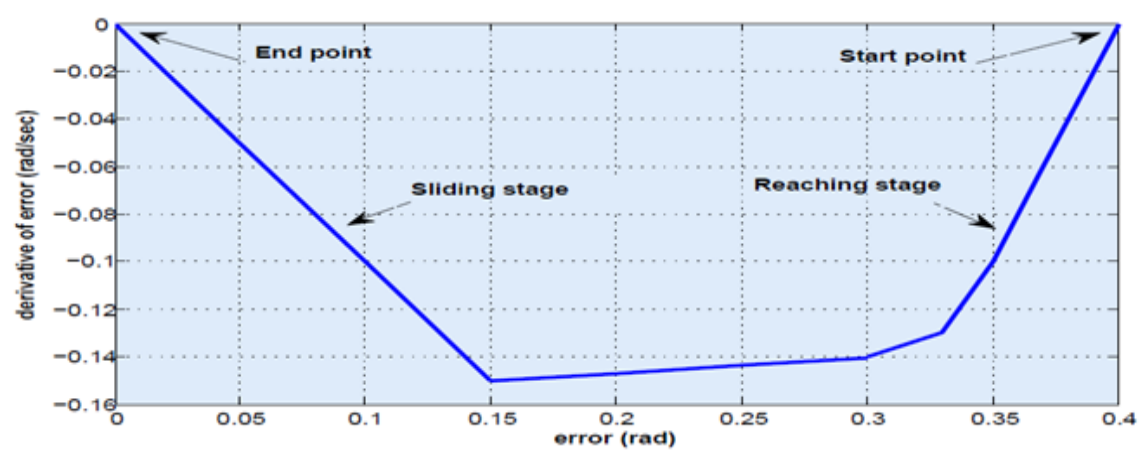

(b)

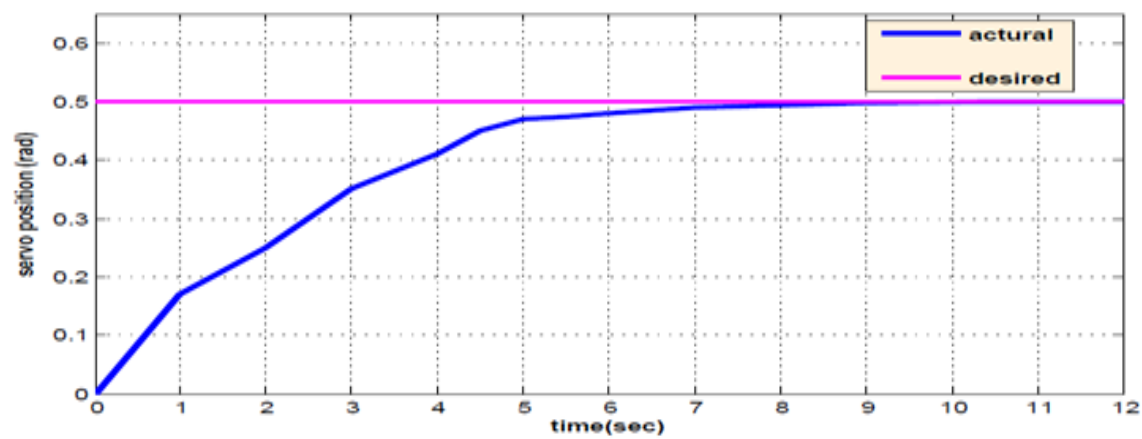

(c)

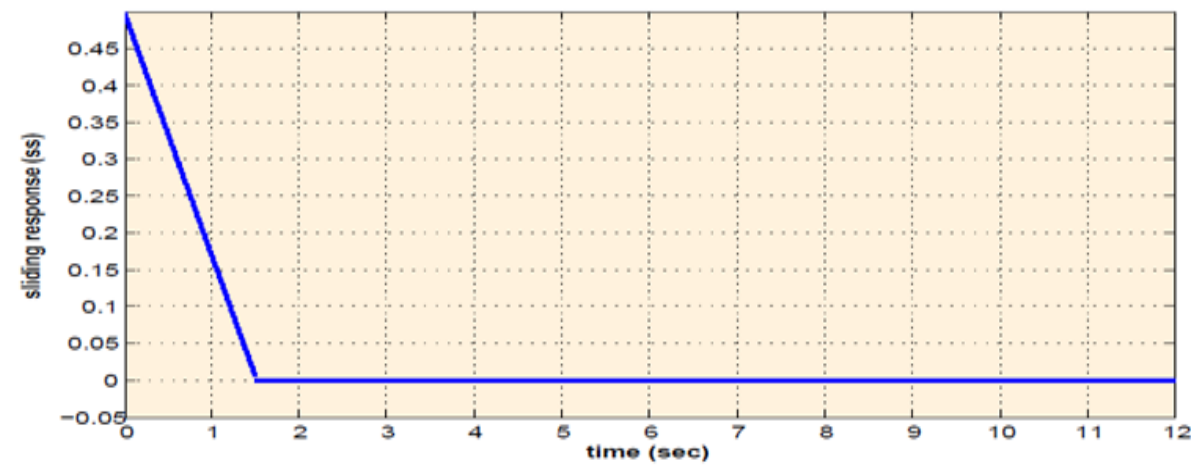

(d)

Figure 8. (a) Control action with sign function, (b) Relationship between error and its derivative with sign function, (c) The actual and desired position with sign function, (d) Sliding response with sign function 


\subsection{CSMCR with sat function}

Similarly, the values as mentioned above with sat function have been illustrated in Figure 9(a)-(d).

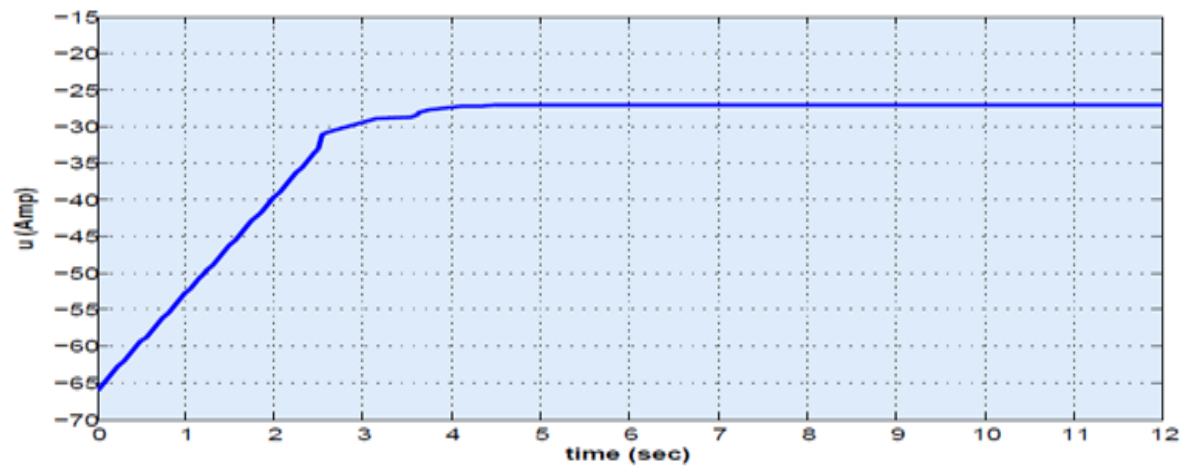

(a)

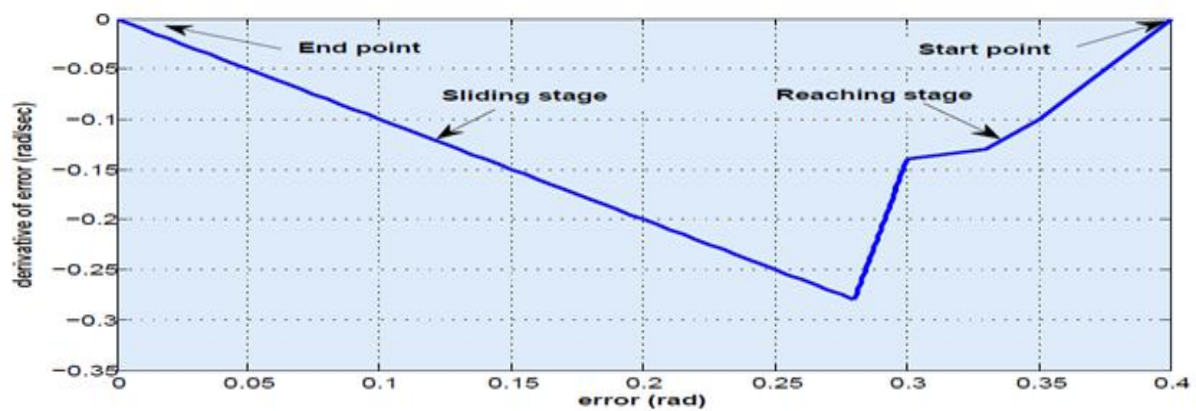

(b)

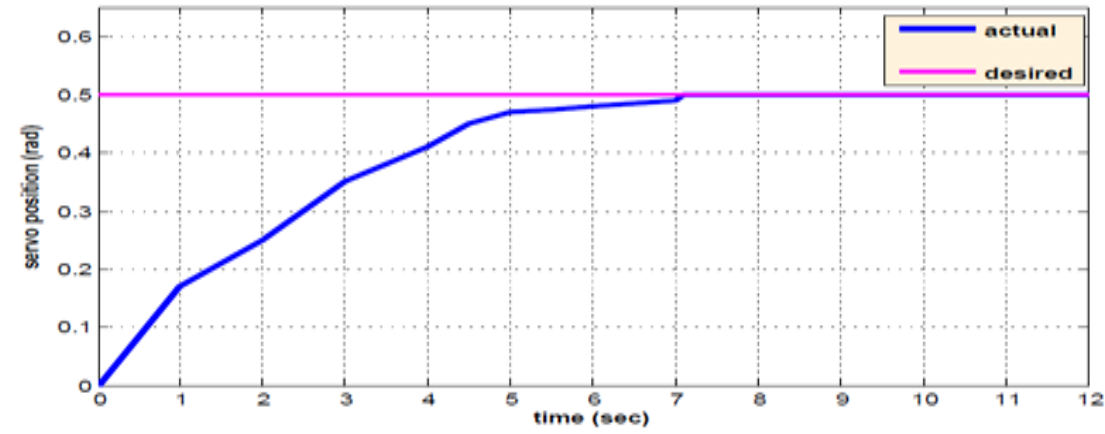

(c)

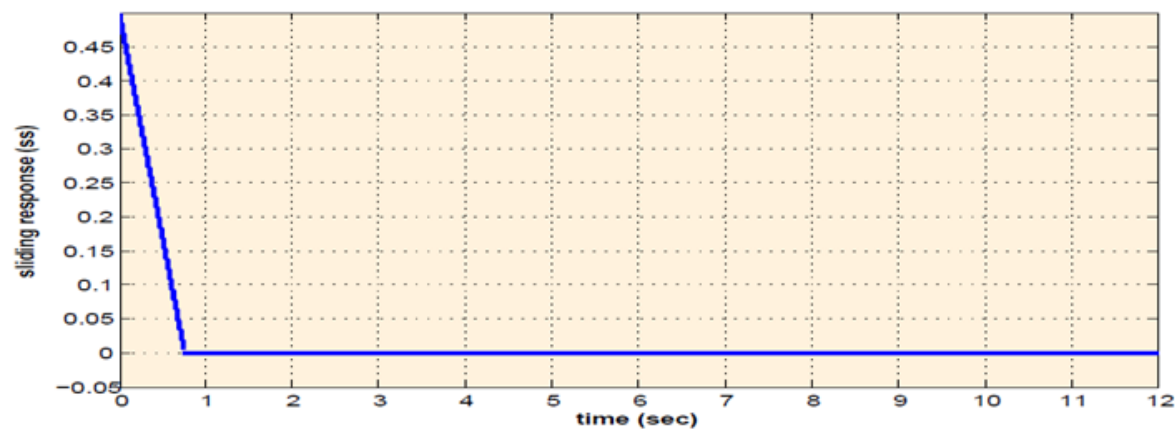

(d)

Figure 9. (a) Control action with sat function, (b) Relationship between error and its derivative with sat function, (c) Actual and desired position with sat function, (d) Sliding response with sat function 


\subsection{CSMCR with sat function and WOA}

In this section, the considerable influence of using WOA with sat function has been shown on the above four criteria of stability of CSMCR, as illustrated in Figure 10(a)-(d).

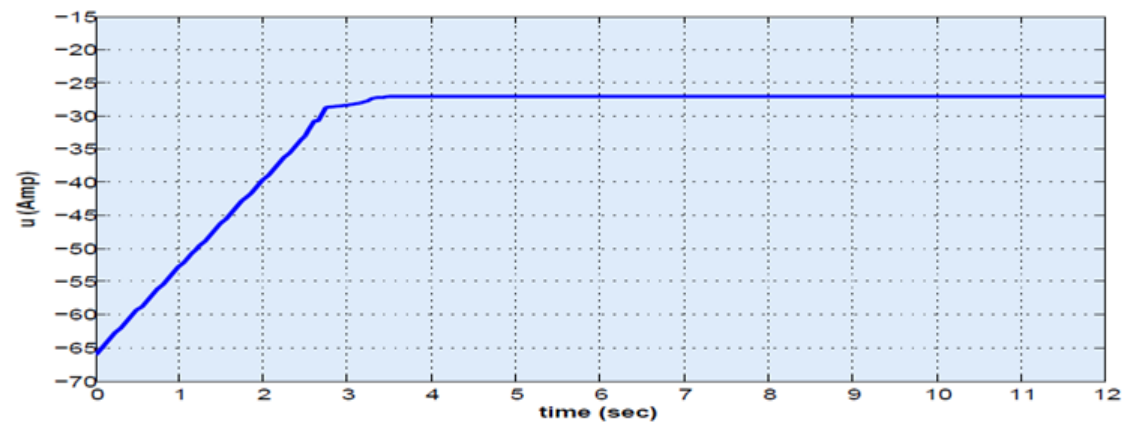

(a)

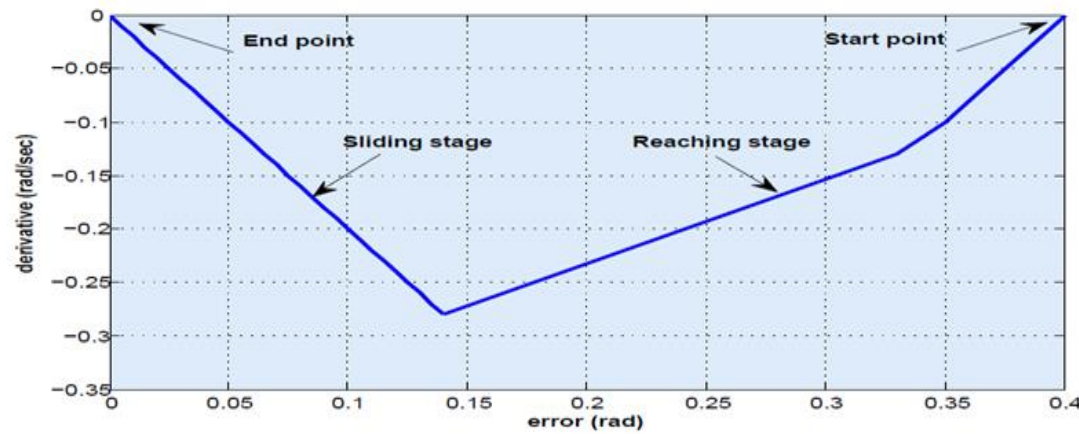

(b)

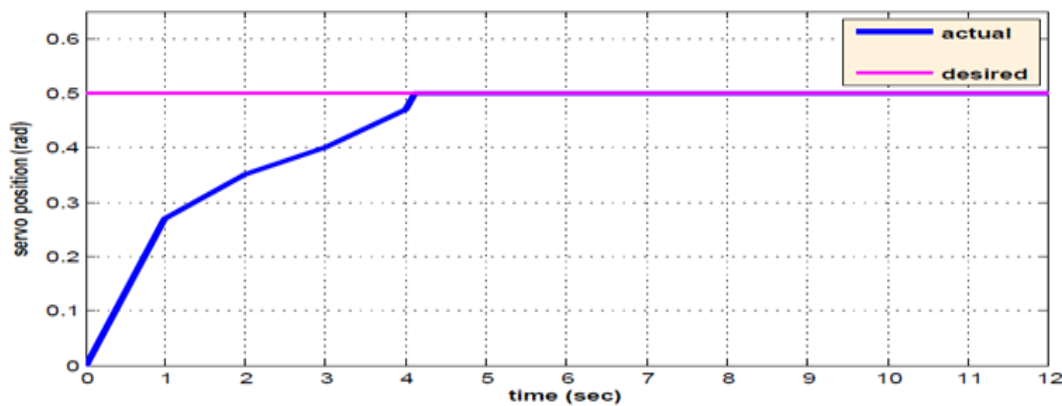

(c)

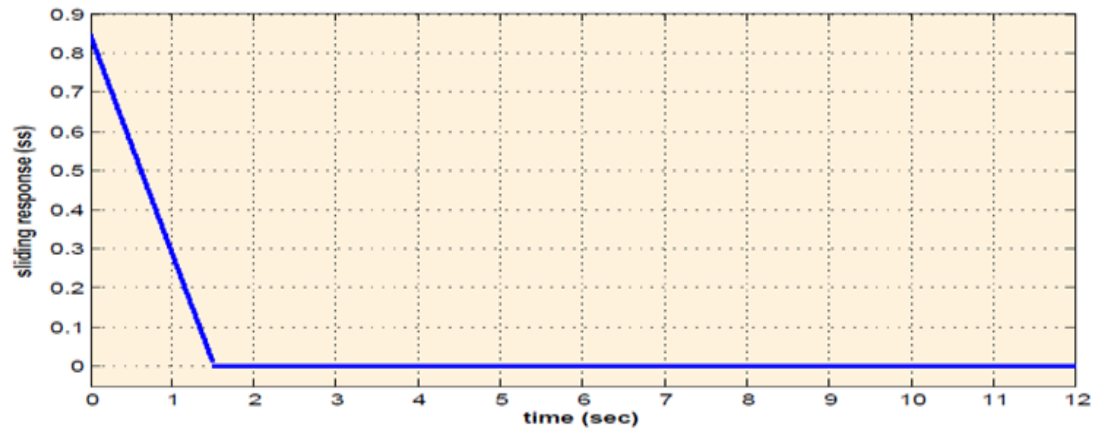

(d)

Figure 10. (a) Control action with sat function and WAO, (b) The relationship between error and its derivative with sat function and WOA, (c) The actual and desired position with sat function and WOA, (d) Sliding response with sat function and WOA 
In terms of control action in three controllers, by comparing Figure 8(a), 9(a), 10(a) a significant improvement is demonstrated by using sat and better with using WOA which contributed in stability maintaining by computing optimal values of gain $\mathrm{G}(\mathrm{x})$ and slope of sliding surface $\delta$ which shown in Table 2 . Furthermore, comparing Figure 8(c), 9(c), 10(c), there is a noticeable reduction in the settling time, as shown in Table 3. Based on the attained results, using WOA has influenced positively on the stability of single inverted pendulum as a nonlinear system.

Table 2. The optimal values of gain $\mathrm{G}(\mathrm{x})$ and slope of sliding surface $\delta$ using WOA

\begin{tabular}{ccc}
\hline Controller & $\mathrm{G}$ & $\Delta$ \\
\hline CSMCR + sign function & 2.873 & 0.987 \\
CSMCR + sat function & 2.541 & 0.964 \\
CSMCR + sat function +WOA & 1.97 & 1.78 \\
\hline
\end{tabular}

Table 3. Settling time of three controllers

\begin{tabular}{cc}
\hline Controller & Settling $(\mathrm{sec})$ \\
\hline CSMCR + sign function & 10.3 \\
CSMCR + sat function & 7.1 \\
CSMCR + sat function +WOA & 4.12 \\
\hline
\end{tabular}

\section{CONCLUSION}

The meta-heuristic optimization algorithm can play a vital role in achieving and keeping the required level of stability. WOA represents a powerful tool in minimizing the chattering problem in CSMCR. The considerable reduction in the settling time as an essential criterion for the system`s stability indicates the reliability of WOA, taking into consideration that the attained outcomes have been computed with limited features of available computer as compared with cutting edge devices.

\section{REFERENCES}

[1] D. A. Zebari, H. Haron, S. R. Zeebaree, and D. Q. Zeebaree, "Multi-Level of DNA Encryption Technique Based on DNA Arithmetic and Biological Operations," in 2018 International Conference on Advanced Science and Engineering (ICOASE), pp. 312-317, 2018.

[2] D. M. Abdulqader, A. M. Abdulazeez, and D. Q. Zeebaree, "Machine Learning Supervised Algorithms of Gene Selection: A Review," Machine Learning, vol. 62, no. 03, 2020.

[3] R. M. Larik, M. W. Mustafa, and M. K. Panjwani, "A statistical jacobian application for power system optimization of voltage stability," Indonesian Journal of Electrical Engineering and Computer Science, vol. 13, no. 1, pp. 331338, 2019.

[4] H. Du, X. Yu, M. Z. Chen, and S. Li, "Chattering-free discrete-time sliding mode control," Automatica, vol. 68, pp. 87-91, 2016.

[5] Y. T. Chen, Y. C. Jhang, and R. H. Liang, "A fuzzy-logic based auto-scaling variable step-size MPPT method for PV systems," Solar Energy, vol. 126, pp. 53-63, 2016.

[6] M. Mir, M. Dayyani, T. Sutikno, M. Mohammadi Zanjireh, and N. Razmjooy, "Employing a Gaussian Particle Swarm Optimization method for tuning Multi Input Multi Output-fuzzy system as an integrated controller of a micro-grid with stability analysis," Computational Intelligence, vol. 36, no. 1, pp. 225-258, 2020.

[7] M. R. Mojallizadeh, M. Badamchizadeh, S. Khanmohammadi, and M. Sabahi, "Designing a new robust sliding mode controller for maximum power point tracking of photovoltaic cells," Solar Energy, vol. 132, pp. 538-546, 2016.

[8] İ. Yazici and E. K. Yaylaci, "Fast and robust voltage control of DC-DC boost converter by using fast terminal sliding mode controller," IET Power Electronics, vol. 9, no. 1, pp. 120-125, 2016.

[9] I. Ethmane, A. Mahmoud, M. Maaroufi, and A. Yahfdhou, "Transient stability enhancement of statcom integration in power grid," Indonesian Journal of Electrical Engineering and Computer Science, vol. 16, no. 2, pp. 553-561, 2019.

[10] C. M. Oliveira, M. L. Aguiar, J. R. Monteiro, W. C. Pereira, G. T. Paula, and T. E. Almeida, "Vector control of induction motor using an integral sliding mode controller with anti-windup," Journal of Control, Automation and Electrical Systems, vol. 27, no. 2, pp. 169-178, 2016.

[11] A. H. Ali, R. F. Chisab, and M. J. Mnati, "A smart monitoring and controlling for agricultural pumps using LoRa IOT technology," Indonesian Journal of Electrical Engineering and Computer Science, vol. 13, no. 1, pp. 286-292, 2019.

[12] N. B. Almutairi and M. Zribi, "Sliding mode controllers for a tempered glass furnace," ISA transactions, vol. 60, pp. 21-37, 2016.

[13] J. B. Valaki and P. P. Rathod, "Assessment of operational feasibility of waste vegetable oil based bio-dielectric fluid for sustainable electric discharge machining (EDM)," The International Journal of Advanced Manufacturing Technology, vol. 87, no. 5-8, pp. 1509-1518, 2016.

[14] B. Sumantri, N. Uchiyama, and S. Sano, "Least square based sliding mode control for a quad-rotor helicopter and energy saving by chattering reduction," Mechanical Systems and Signal Processing, vol. 66, pp. 769-784, 2016.

[15] N. Mamat, F. Yakub, S. Salim, and M. S. M. Ali, "Performance Comparison of Controllers for Suppressing the Structural Building Vibration," Indonesian Journal of Electrical Engineering and Computer Science, vol. 10, no. 2, pp. 537-544, 2018. 
[16] J. Zhao, T. Li, and J. Qian, "Application of particle swarm optimization algorithm on robust PID controller tuning," in International Conference on Natural Computation, 2005, pp. 948-957.

[17] S. A. Al-Samarraie, "Invariant sets in sliding mode control theory with application to servo actuator system with friction," Wseas transactions on systems and control, vol. 8, no. 2, pp. 33-45, 2013.

[18] F. Plestan, Y. Shtessel, V. Brégeault, and A. Poznyak, "Sliding mode control with gain adaptation -Application to an electropneumatic actuator," Control Engineering Practice, vol. 21, no. 5, pp. 679-688, 2013.

[19] C. Zhou, X. Liu, W. Chen, F. Xu, and B. Cao, "Optimal sliding mode control for an active suspension system based on a genetic algorithm," Algorithms, vol. 11, no. 12, p. 205, 2018.

[20] N. Bounar, S. Labdai, and A. Boulkroune, "PSO-GSA based fuzzy sliding mode controller for DFIG-based wind turbine," ISA transactions, vol. 85, pp. 177-188, 2019.

[21] A. T. S. Al-Obaidi, H. S. Abdullah, and Z. O. Ahmed, "Meerkat clan algorithm: A new swarm intelligence algorithm," Indonesian Journal of Electrical Engineering and Computer Science, vol. 10, no. 1, pp. 354-360, 2018.

[22] M. K. M. Zamani et al., "Active and reactive power scheduling optimization using firefly algorithm to improve voltage stability under load demand variation," Indonesian Journal of Electrical Engineering and Computer Science, vol. 9, no. 2, pp. 365-372, 2018.

[23] D. P. Adhyapak, B. Sridharan, and A. P. Laturkar, "Swarm based cross layer optimization protocol for WMSN," Indonesian Journal of Electrical Engineering and Computer Science, vol. 10, no. 1, pp. 302-308, 2018.

[24] M. F. Hamza, H. J. Yap, I. A. Choudhury, A. I. Isa, A. Y. Zimit, and T. Kumbasar, "Current development on using Rotary Inverted Pendulum as a benchmark for testing linear and nonlinear control algorithms," Mechanical Systems and Signal Processing, vol. 116, pp. 347-369, 2019.

[25] J. A. Goldbogen, A. S. Friedlaender, J. Calambokidis, M. F. Mckenna, M. Simon, and D. P. Nowacek, "Integrative approaches to the study of baleen whale diving behavior, feeding performance, and foraging ecology," BioScience, vol. 63, no. 2, pp. 90-100, 2013.

[26] A. S. Eesa, A. M. A. Brifcani, and Z. Orman, "Cuttlefish algorithm-a novel bio-inspired optimization algorithm," International Journal of Scientific \& Engineering Research, vol. 4, no. 9, pp. 1978-1986, 2013.

[27] K. Umapathy and N. Khare, "An Efficient \& Secure Content Contribution and Retrieval Content in Online Social Networks Using Level-level Security Optimization \& Content Visualization Algorithm,” Indonesian Journal of Electrical Engineering and Computer Science, vol. 10, no. 2, pp. 807-816, 2018.

[28] A. S. Eesa, A. M. A. Brifcani, and Z. Orman, "A new tool for global optimization problems-cuttlefish algorithm," International Journal of Mathematical, Computational, Natural and Physical Engineering, vol. 8, no. 9, pp. 1208-1211, 2014.

[29] A. S. Eesa, Z. Orman, and A. M. A. Brifcani, "A novel feature-selection approach based on the cuttlefish optimization algorithm for intrusion detection systems," Expert Systems with Applications, vol. 42, no. 5, pp. 2670-2679, 2015.

[30] S. Mirjalili and A. Lewis, "The whale optimization algorithm," Advances in engineering software, vol. 95, pp. 51-67, 2016.

[31] J. Wang, P. Du, T. Niu, and W. Yang, "A novel hybrid system based on a new proposed algorithm-MultiObjective Whale Optimization Algorithm for wind speed forecasting," Applied energy, vol. 208, pp. 344-360, 2017. 